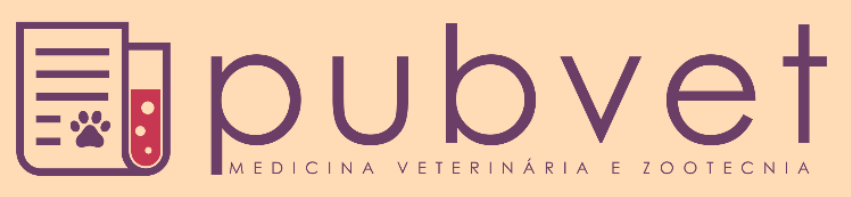

https://doi.org/10.31533/pubvet.v15n08a880.1-5

\title{
Avaliação comparativa entre dois glicosímetros portáteis e o método laboratorial enzimático colorimétrico na dosagem glicêmica em cavalos
}

\author{
Karin Heloise Tavares $^{1 \bullet}$, Pricilla Pozzatti ${ }^{2 *}$, Carlos Eduardo Nogueira Martins ${ }^{2} \bullet$ \\ ${ }^{I}$ Graduanda em medicina veterinária no Instituto Federal Catarinense. Araquari - SC. Brasil \\ ${ }^{2}$ Docente do curso de Medicina Veterinária do Instituto Federal Catarinense-Campus Araquari-SC. Brasil \\ *Autor para correspondência: pricilla.pozzatti@ifc.edu.br
}

\begin{abstract}
Resumo. Na clínica equina, o sistema de aferição de referência para glicemia é o método laboratorial enzimático colorimétrico. Entretanto, é necessário equipamento, reagente específico e maior demanda de tempo para sua realização. Devido a praticidade, os glicosímetros portáteis vêm ganhando espaço na medicina veterinária, fornecendo o resultado de forma rápida, facilitando a dosagem da glicemia a campo. Diversos estudos utilizaram glicosímetros portáteis para aferição da glicemia em equinos, contudo, ainda há muitas controvérsias sobre a confiabilidade de seus resultados em comparação com o método enzimático. Assim sendo, o objetivo deste trabalho foi avaliar a utilização de dois glicosímetros portáteis para a mensuração da glicemia em equinos considerando como controle o teste laboratorial convencional. Para tanto, foram avaliados o glicosímetro portátil veterinário Wellion GLUCO CALEA ${ }^{\circledR}$ e o glicosímetro portátil humano ACCUCHEK Performa ${ }^{\circledR}$ e comparados com o teste enzimático colorimétrico. Concluiu-se que o glicosímetro ACCU-CHEK Performa ${ }^{\circledR}$ pode ser utilizado para testes de glicemia rápida em equinos.
\end{abstract}

Palavras-chave: Equinos, glicemia, glicose, laboratorial

\section{Comparative evaluation between two portable glucometers and the enzymatic colorimetric laboratory method in glycemic dosage in horses}

\begin{abstract}
In the equine clinic, the reference system for measuring blood glucose is the colorimetric enzymatic laboratory method. However, equipment, specific reagent and more time are required to perform it. Due to practicality, portable glucometers have been gaining ground in veterinary medicine, providing the result quickly, facilitating the measurement of blood glucose in the field. Several studies have used portable glucometers to measure blood glucose in horses, however, there are still many controversies about the reliability of their results compared to the enzymatic method. Therefore, the objective of this work was to evaluate the use of two portable glucometers for the measurement of blood glucose in horses considering the conventional laboratory test as control. For this purpose, the Wellion GLUCO CALEA ${ }^{\circledR}$ portable veterinary glucometer and the ACCU-CHEK Performa ${ }^{\circledR}$ human portable glucometer were evaluated and compared with the colorimetric enzymatic test. It was concluded that the ACCUCHEK Performa ${ }^{\circledR}$ glucometer can be used for rapid blood glucose tests in horses.
\end{abstract}

Keywords: Blood glucose, equine, glucose, laboratory 


\section{Evaluación comparativa entre dos glucómetros portátiles y el método de laboratorio enzimático colorimétrico en la dosis glicémica de caballos}

Resumen. En la clínica equina, el sistema de exploración de referencia para la glicemia es el método de laboratorio enzimático colorimétrico. Sin embargo, se necesita de equipos, reactivos específicos y mayor demanda de tiempo para su realización. Debido a su practicidad, los glucómetros portátiles vienen ganando espacio en la Medicina Veterinaria, ofreciendo el resultado de forma rápida, facilitando la dosis de la glucosa en sangre a campo. Diferentes estudios utilizaran glucómetros portátiles para la medición de la glucosa en equino, sin embargo, aún existen muchas controversias sobre la confiabilidad de los resultados en comparación con el método enzimático. De esta manera, El objetivo de este trabajo fue evaluar la utilización de dos glucómetros portátiles para la medición de la glucosa en sangre en equinos considerando como control la prueba de laboratorio convencional. Fueron evaluados el glucómetro portátil veterinario Wellion GLUCO CALEA ${ }^{\circledR}$ y el glucómetro portátil humano ACCU-CHEK Performa ${ }^{\circledR}$ y comparados con la prueba enzimática colorimétrica. Se concluye que el glucometro ACCU-CHEK Performa ${ }^{\circledR}$ puede ser utilizado para realizar pruebas de glicemia rápida en equinos.

Palavras clave: Equinos, glucosa em sangre, glucosa, laboratorio

\section{Introdução}

A aferição dos níveis glicêmicos em cavalos auxilia na rotina da clínica médica como um preditivo de complicações sistêmicas, auxiliando no acompanhamento do estado metabólico e no diagnóstico de enfermidades (Hollis et al., 2007). Hollis et al. (2007) avaliaram registros de cavalos com síndrome do abdômen agudo e observou que 50,2\% sofriam de hiperglicemia. A confirmação obtida por Hassel et al. (2009) de que a hiperglicemia está relacionada com um prognóstico desfavorável em cavalos com síndrome cólica, ressalta a importância da realização do controle da glicemia na terapia intensiva. Todavia, a mensuração glicêmica em hospitais geralmente é feita pelo método laboratorial, não sendo viável para veterinários que realizam atendimento emergencial a campo, tendo como alternativa, a utilização de glicosímetros portáteis (Hackett \& McCue, 2010). Pesquisas com resultados favoráveis a utilização desses glicosímetros para equinos foram relatadas, como o estudo realizado por Hackett \& McCue (2010), onde avaliaram o glicosímetro veterinário portátil (GVP) encontrando precisão nos resultados quando comparados com a análise laboratorial. Outro resultado foi o obtido por Kittle et al. (2019) que avaliaram o glicosímetro portátil humano (GPH), obtendo resultado favorável das mensurações. Entretanto, Teixeira Neto et al. (2011) relatam que os glicosímetros portáteis humanos não são ideais para avaliação glicêmica quando comparando com o teste padrão. Frente às dúvidas levantadas pela grande quantidade de estudos existentes e a quantidade elevada de marcas de aparelhos portáteis presentes no mercado, pode haver o risco de interpretação errônea dos valores glicêmicos, influenciando na conduta clínica do médico veterinário. Assim, a relevância clínica da monitoração da glicemia em cavalos que necessitem de cuidados intensivos em hospitais ou a campo, aumenta diretamente a necessidade de métodos de mensuração glicêmica rápidos associados à acurácia em resultados.

\section{Material e métodos}

O presente estudo foi submetido e autorizado pelo Comitê de Ética na Utilização de Animais (CEUA). Para a sua realização foram utilizados 16 equinos sem restrição de raça, sexo e/ou idade, pertencentes a propriedades particulares situadas no município de Araquari, região norte do estado de Santa Catarina. Para obtenção das amostras foram utilizadas agulhas de calibre 30x8G e seringa de 5 $\mathrm{mL}$. A coleta foi realizada através da venopunção da jugular, de maneira que foram acondicionados $2,5 \mathrm{~mL}$ em tubos Vacutainer ${ }^{\circledR}$ contendo Fluoreto de Sódio e o restante foi diretamente utilizado para mensuração da glicemia nos aparelhos portáteis ACCU-CHEK Performa ${ }^{\circledR}$ e Wellion GLUCO CALEA $^{\circledR}$. As amostras foram encaminhadas para o Laboratório de Patologia Clínica Veterinária do 
IFC - Campus Araquari, onde foram centrifugadas a 3000 RPM durante 10 minutos na centrífuga DAIKI $80-2 \mathrm{~B}^{\circledR}$ para a obtenção do plasma.

Para a análise da concentração de glicose plasmática foi utilizado o reagente Labtest Glicose Liquiform $\mathrm{Vet}^{\circledR}$ e analisador bioquímico MAX BIO $\mathrm{TOUCH}^{\circledR}$. Cada amostra foi avaliada em duplicata, para verificação da confiabilidade do aparelho e do reagente com posterior cálculo da média para análises estatísticas. Todos os resultados foram expressos em $\mathrm{mg} / \mathrm{dL}$ e o teste estatístico realizado foi o teste de normalidade de Shapiro-Wilk. Como os resultados obtidos nesta primeira etapa estatística não mostraram distribuição normal, foi também realizado o teste não paramétrico de significância estatística de Kruskal-Wallis para comparação dos resultados e, posteriormente, o teste Dwass-Steel-Critchlow-Fligner (DSCF) de comparação de médias.

\section{Resultados e discussão}

Os resultados obtidos pela coleta de sangue para comparação entre os glicosímetros portáteis com o exame laboratorial se encontram expressos na tabela 1.

Tabela 1. Resultados obtidos na determinação da glicose por meio de teste laboratorial e pelos glicosímetros portáteis Wellion GLUCO CALEA e ACCU-CHEK Performa em mg/dL.

\begin{tabular}{lccc}
\hline Animal & Teste laboratorial $^{\text {a }}$ & ACCU-CHECK Performa $^{\circledR a}$ & Wellion GLUCO CALEA $^{\oplus b}$ \\
1 & 78 & 79 & 121 \\
2 & 85 & 78 & 17 \\
3 & 80 & 97 & 102 \\
4 & 91 & 98 & 130 \\
5 & 80 & 89 & 126 \\
6 & 87 & 92 & 114 \\
7 & 82 & 92 & 80 \\
9 & 76 & 61 & 115 \\
10 & 74 & 73 & 125 \\
11 & 75 & 90 & 140 \\
12 & 82 & 98 & 121 \\
13 & 72 & 84 & 127 \\
14 & 76 & 89 & 118 \\
16 & 93 & 90 & 125 \\
\hline
\end{tabular}

Letras sobrescritas iguais representam semelhança estatística $(\mathrm{p}<0.01)$, enquanto letras sobrescritas diferentes significam diferença de resultados $(\mathrm{P}=0,064)$.

Os valores mínimos e máximos encontrados nas amostras analisadas pelo teste laboratorial foram de 72 e $93 \mathrm{mg} / \mathrm{dL}$. Os valores obtidos pelo glicosímetro ACCU-CHEK Performa ${ }^{\circledR}$ foram 61 e 98 $\mathrm{mg} / \mathrm{dL}$ onde somente a amostra coletada do animal número 8 apresentou resultado abaixo do intervalo de referência citado por Thrall et al. (2014) que é de 70 à $110 \mathrm{mg} / \mathrm{dL}$, enquanto os valores do glicosímetro Wellion GLUCO CALEA ${ }^{\circledR}$ variaram entre 77 e $140 \mathrm{mg} / \mathrm{dL}$, sendo que $81,25 \%$ dos resultados deste último, encontraram-se acima do mesmo intervalo citado. Essa alteração não interfere no objetivo deste estudo que é o da comparação entre as metodologias e verificação sobre qual glicosímetro pode ser adotado na rotina clínica.

A partir destes resultados, foram calculados os valores médios e os desvios padrão (DP) dos valores de glicose para cada método avaliativo, tendo glicosímetro portátil veterinário Wellion GLUCO CALEA $^{\circledR}$ a média de $117 \mathrm{mg} / \mathrm{dL}$ e DP de 4,30), o glicosímetro portátil ACCU-CHEK Performa ${ }^{\circledR}$ a média de $86,1 \mathrm{mg} / \mathrm{dL}$ e DP de 2,46 e teste laboratorial média de 79,9 mg/dL e DP de 1,60.

Para o glicosímetro Wellion GLUCO CALEA ${ }^{\circledR}$ em seu manual de instrução não há especificação do seu intervalo de referência para a espécie equina, indicando a decisão ao médico veterinário. Com base nisso, se compará-lo aos resultados obtidos pelo teste laboratorial padrão ouro para a análise, que se encontra dentro dos limites estabelecidos por Thrall (2015) e pelas instruções de uso contidas no manual LABTEST Glicose Liquiform Vet ${ }^{\circledR}(70 \mathrm{a} 110 \mathrm{mg} / \mathrm{dL})$ é visto que a média de seus resultados apresenta valor fora do intervalo de referência utilizado para a análise dos outros métodos, concordando com estudos anteriores realizados por Hackett \& McCue (2010), inviabilizando a sua 
utilização, visto o risco de resultados errôneos que podem culminar num falso indicativo de hiperglicemia e complicações sistêmicas graves (Nasraway Junior, 2006).

Tendo em vista os valores dos resultados encontrados pela mensuração da glicose via glicosímetro Wellion GLUCO CALEA ${ }^{\circledR}$, a estatística confirmou a diferença entre os valores obtidos nos testes entre este glicosímetro e o método enzimático colorimétrico usado no presente trabalho. Hollis et al. (2008) e Teixeira Neto et al. (2011) já haviam verificado a inviabilidade da utilização de glicosímetros portáteis como Accutrend ${ }^{\circledR}$ Plus e o Accu-Chek ${ }^{\circledR}$ Aviva, corroborando com o resultado obtido pelo deste trabalho com a utilização do glicosímetro Wellion GLUCO CALEA ${ }^{\circledR}$.

Quando se comparou os resultados do glicosímetro portátil humano ACCU-CHEK Performa ${ }^{\circledR}$ com o teste enzimático colorimétrico não houve diferença significativa, concordando em partes com por Bromerschenkel et al. (2015) e Kittle et al. (2019) que disseram que a utilização de glicosímetros de uso humano é seguro e que seus resultados podem ser comparados aos métodos enzimáticos.

Os resultados obtidos pelo glicosímetro veterinário Wellion GLUCO CALEA® não devem ser comparados aos do glicosímetro ACCU-CHEK Performa ${ }^{\circledR}$, visto que esse tem seus resultados não diferentes dos encontrados pelo teste laboratorial, podendo ser analisados com base no intervalo fornecido por Thrall (2015). Isso faz com que o uso do glicosímetro Wellion GLUCO CALEA ${ }^{\circledR}$ seja questionável, por assim como Hollis et al. (2008), o presente estudo ter encontrado diferença dos resultados quando comparado ao teste laboratorial. Entretanto, tendo o atual estudo obtido resultados utilizando o glicosímetro ACCU-CHEK Performa ${ }^{\circledR}$, corrobora com diversos autores como Bromerschenkel et al. (2015), Hollis et al. (2008) e Kittle et al. (2019) que concordam entre si de que o uso do glicosímetro portátil humano pode ser indicado para amensuração da glicose de equinos.

\section{Considerações finais}

O glicosímetro portátil ACCU-CHEK Performa ${ }^{\circledR}$ de utilização humana pode ser utilizado na clínica de equinos para mensuração da glicemia de forma rápida.

O glicosímetro portátil Wellion GLUCO CALEA ${ }^{\circledR}$ de uso exclusivo veterinário só deve ser utilizado para cavalos quando for adotado o intervalo de referência estabelecido pelo próprio fabricante.

Não se deve comparar o resultado do glicosímetro portátil Wellion GLUCO CALEA $^{\circledR}$ com o glicosímetro ACCU-CHEK Performa ${ }^{\circledR}$ e nem testes laboratoriais.

\section{Referências}

Bromerschenkel, I., Porfírio, L. C., \& Martins, C. B. (2015). Uso do glicosímetro portátil para a mensuração da glicemia em potros neonatos da raça mangalarga marchador. Acta Veterinaria Brasilica, 9(4), 369-374. DOI: https://doi.org/10.21708/avb.2015.9.4.5437

Hackett, E. S., \& McCue, P. M. (2010). Evaluation of a veterinary glucometer for use in horses. Journal of Veterinary Internal Medicine, 24(3), 617-621. DOI: https://doi.org/10.1111/j.1939$\underline{1676.2010 .0481 . \mathrm{x}}$

Hassel, D. M., Hill, A. E., \& Rorabeck, R. A. (2009). Association between hyperglycemia and survival in 228 horses with acute gastrointestinal disease. Journal of Veterinary Internal Medicine, 23(6), 1261-1265. DOI: https://doi.org/10.1111/j.1939-1676.2009.0395.x

Hollis, A. R., Boston, R. C., \& Corley, K. T. T. (2007). Blood glucose in horses with acute abdominal disease. Journal of Veterinary Internal Medicine, 21(5), 1099-1103. DOI: https://doi.org/10.1111/j.1939-1676.2007.tb03070.x

Hollis, A. R., Furr, M. O., Magdesian, K. G., Axon, J. E., Ludlow, V., Boston, R. C., \& Corley, K. T. T. (2008). Blood glucose concentrations in critically ill neonatal foals. Journal of Veterinary Internal Medicine, 22(5), 1223-1227. DOI: https://doi.org/10.1111/j.1939-1676.2008.0174.x

Kittle, A. J., Sisler, S. E., Nguyen, N., Gill, J. C., \& Reed, V. (2019). Method-comparison analysis between a Contour Next glucometer and YSI 2900 biochemistry analyzer in equine. Journal of Equine Veterinary Science, 76, 127. DOI: https://doi.org/10.1016/j.jevs.2019.03.203 
Labtest diagnósticos S.A. (2012). Labtest Glicose Liquiform Vet®: Instruções de uso. Vista Alegre: labtest, 2012. 6p.

Nasraway Junior, S. A. (2006). Hyperglycemia during critical illness. Journal of Parenteral and Enteral Nutrition, 30(3), 254-258. DOI: https://doi.org/10.1177/0148607106030003254

Teixeira Neto, A. R., Botelho, R. G., Sousa, K. E., Gomes, J. L., \& Lima, E. M. M. (2011). Ineficácia do aparelho portátil accutrend plus na clínica médica de eqüinos. ARS Veterinaria, 27, 17-21.

Thrall, M. A. (2015). Hematologia e Bioquímica Clínica Veterinária. In 2. ed. Editora Roca.

Histórico do artigo:

Recebido: 10 de março de 2021

Aprovado: 27 de abril de 2021.
Licenciamento: Este artigo é publicado na modalidade Acesso Aberto sob a licença Creative Commons Atribuição 4.0 (CC-BY 4.0), a qual permite uso irrestrito, distribuição, reprodução em qualquer meio, desde que $\mathrm{o}$ autor $\mathrm{e}$ a fonte sejam devidamente creditados. 